\title{
Biomimetic vascularized adipose-derived mesenchymal stem cells bone-periosteum graft enhances angiogenesis and osteogenesis in a rabbit spine fusion model
}

\section{Tsai-Sheng Fu ( $\nabla$ fts111@adm.cgmh.org.tw) \\ Chang Gung Memorial Hospital Keelung Branch \\ Wei-Chuan Chen \\ Yuan Ze University \\ Ying-Chih Wang \\ Chang Gung Medical College: Chang Gung University \\ Chia-Wei Chang \\ Chang Gung Medical College: Chang Gung University \\ Tung-Yi Lin \\ Chang Gung Medical College: Chang Gung University \\ Chak-Bor Wong \\ Chang Gung Medical College: Chang Gung University}

\section{Research}

Keywords: Tissue engineering, Cell sheet, Osteogenesis, Angiogenesis, Biomimetic bone-periosteum graft, Adipose-derived mesenchymal stem cell

Posted Date: September 15th, 2021

DOI: https://doi.org/10.21203/rs.3.rs-880132/v1

License: (c) (1) This work is licensed under a Creative Commons Attribution 4.0 International License. Read Full License 


\section{Abstract}

\section{Background}

Several artificial bone grafts have been developed for bone reconstruction but fail to achieve anticipated osteogenesis due to their insufficient neovascularization capacity. Besides, periosteum plays an essential role in the neovascularization process in bone formation and healing. The aim of this study was to develop a cell-based biomimetic vascularized bone-periosteum construct (VBPC) to provide better neovascularization for osteogenesis and bone regeneration.

Methods

Twenty-four male New Zealand white rabbits were divided into four groups according to the experimental materials. We first cultured adipose-derived mesenchymal stem cells (AMSCs) and seeded them evenly in the collagen/chitosan sheet to form an AMSCs-sheet-engineered periosteum. Simultaneously, the AMSCs were seeded onto alginate scaffolds and were cultured to differentiate to endothelial-like cells to form vascularized bone constructs (VBC). The success of endothelial differentiation was confirmed by realtime polymerase chain reaction and immunofluorescence staining analysis. The AMSCs-sheet-engineered periosteum was wrapped onto VBC to create biomimetic VBPC, which was then implanted in bilateral L45 intertransverse space of rabbit. The acellular alginate-sheet construct, VBC, and non-vascularized AMSCs-alginate-periosteum construct were used as controls. At 12 weeks after implantation, the boneforming capacities of the constructs were determined by computed tomography, biomechanical testing, histology, and immunohistochemistry staining analyses.

\section{Results}

Twelve weeks after implantation, the VBPC group significantly increased new bone formation volume than the control groups. Biomechanical testing demonstrated a higher torque strength in the VBPC group, and suggested that the cell sheet played a critical role for mechanical support. Notably, the hematoxylin and eosin, Masson's trichrome, and immunohistochemistry stained histologic results revealed that the VBPC group promoted the formation of blood vessels and new bones in the L4-5 intertransverse fusion areas.

\section{Conclusions}

The tissue-engineered biomimetic VBPC showed great capability in promoting angiogenesis and osteogenesis in vivo. The VBPC may overcome the deficits of traditional bone grafts. These findings suggest a novel approach to improve the timely formation of blood vessels from bone substitutes and provide an ideal source for bone regeneration.

\section{Introduction}


With the coming of population aging, degenerative spinal disorders such as spondylolisthesis with spinal stenosis and osteoporotic vertebral fractures become more and more common [1]. Spinal fusion surgery is performed increasingly. Using autologous bone graft is the gold standard for spinal fusion surgery owing to several critical factors: (1) a bony scaffold, (2) rich in growth factors in extracellular matrix, and (3) sufficient osteoprogenitor cells from bone marrow [2]. However, autologous bone graft has its own disadvantages, including donor-side morbidity and increased operation time, which has led to the development of alternative graft materials [3]. In addition, current synthetic bone grafts have failed to achieve anticipated fusion rates owing to their insufficient vascularization capacity for new bone formation [4].

Recently, the mesenchymal stem cells (MSCs) from bone marrow have been used to fabricate tissueengineered graft for bone healing [5,6]. However, the insufficient number of cells from bone marrow reduces the potential for clinical use. Adipose-derived mesenchymal stem cells (AMSCs) obtained from adipose tissue has been paid attention to its potential for clinical use owing to large number of cells [7]. The AMSCs can proliferate rapidly and maintain their differential potential such as osteogenesis and angiogenesis cells, which is a good source of stem cells for bone tissue engineering [7]. Moreover, AMSCs secrete growth factors such as fibroblast growth factor-2 (FGF-2) and vascular endothelial growth factor (VEGF), which can stimulate new blood vessels formation and are extremely important for bone formation [8].

From the structural perspective, the cortical bone is covered by a periosteum on its outer surface. The periosteum plays a crucial role in bone development and fracture healing. Reports demonstrated that periosteum not only provides skeletal stem/progenitor cells for fracture healing, but also affects cell cytoskeletal reorganization [9-11]. For bony tissue engineering, a biodegradable three-dimension (3D) scaffold is critical as a matrix for cellular adhesion, growth, differentiation and proliferation to produce bone without eliciting inflammatory reactions. The matrix of native bone largely comprises of type I collagen, which is extensively used in bone tissue engineering owing to its excellent osteoconductivity for cell proliferation and osteogenic differentiation [5-7]. Currently, cell sheet engineering using MSCs-based preparation techniques for regenerative medicine has revealed great potential in bone healing [12-14]. Thus, tissue engineering approach using a periosteum-mimetic cell sheet to wrap a structural cellimpregnated scaffold might create a biomimetic bone-periosteum construct as native bone theoretically.

Chitosan (D-glucosamine linked to $\mathrm{N}$-acetyl D-glucosamine by $\beta$-1,4-glycosidic bond) produced commercially via the deacetylation of chitin is the main structural component of the crustacean exoskeleton and is used as a film, fiber and porous scaffold $[15,16]$. Alginate is a natural anionic biopolymer and has antioxidant and anti-inflammatory properties for a wide variety of applications, such as wound dressing materials, 3D culture, cell and protein delivery, and cardiac regeneration [17-19]. Combination of chitosan and alginate may produce an ideal biocompatible and biodegradable 3D framework as a cell carrier scaffold. In the current study, the authors create a biomimetic vascularized bone-periosteum construct (VBPC) by using an engineered biomimetic periosteum to wrap around the alginate beads seeded with epithelial-differentiated AMSCs. It may provide a novel approach to overcome 
the deficits of insufficient vascularization capacity of current artificial bone graft and to create a superior nutritional environment for cell growth and osteogenesis. Our data demonstrated that the novel biomimetic VBPC improves vascularization and bone regeneration using a New Zealand white rabbit spine fusion model in vivo.

\section{Materials And Methods}

Male New Zealand white rabbits weighing 3.5 to $4 \mathrm{~kg}$ were used in the study. Approval was obtained from the Institutional Animal Care and Use Committee at the authors' institute before the study (permit number: 2015121201).

Preparation of rabbit adipose-derived mesenchymal stem cells (AMSCs).

Adipose tissue collected from New Zealand white rabbits was immersed in 2\% PSA (Thermo Fisher Scientific, Waltham, MA) in PBS (Sigma-Aldrich, St Louis, MO) and washed twice in PBS by centrifugation. The blood vessels in the adipose tissue were cleaned to remove blood from the tissue. Then, cut them into small pieces and placed in a $0.075 \%$ collagenase type I solution for 60 minutes at $37^{\circ} \mathrm{C}$. The collagenase activity was neutralized with $10 \%$ FBS (Hyclone, Logan, UT) in low-glucose DMEM (Gibco-BRL, Carlsbad, CA) and centrifuged at $1200 \mathrm{rpm}$ for 10 minutes. Aspirated the supernatant and dissolved with PBS. Filter excess tissue with 100um nylon mash. The filtrate was centrifuged at $1200 \mathrm{rpm}$ for 10 minutes and removed the supernatant. The cells were reconstituted with $20 \% \mathrm{FBS}, 100 \mathrm{U} / \mathrm{mL}$ penicillin-streptomycin (Hyclone) in low-glucose DMEM, and cultured at $37^{\circ} \mathrm{C}$ in $5 \% \mathrm{CO}_{2}$ incubator.

Flow cytometric analysis of AMSCs.

After 14-day cultivation, the AMSCs (passage 3) were harvested using $0.25 \%$ trypsin/EDTA. A $100-\mu \mathrm{L}$ cell suspension with a cell density of $5 \times 10^{5} / \mathrm{mL}$ was transferred into an Eppendorf tube and then incubated with the following specific fluorescein isothiocyanate (FITC) conjugated monoclonal antibodies, including CD9 (1:200, AbD Serotec Ltd., Oxford, UK), CD29 (1:200, MilliporeSigma, Burlington, MA), CD44 (1:200, AbD Serotec Ltd.), CD73 (1:200, eBioScience, San Diego, CA), CD90 (1:200, BioLegend, San Diego, CA), CD105 (1:200, Biorbyt, Cambridge, UK), CD 45 (1:200, Thermo Fisher Scientific, Waltham, MA) and CD34 $(1: 200$, GeneTex, Irvine, $\mathrm{CA})$ for $20 \mathrm{~min}$ at $4^{\circ} \mathrm{C}$. Then the stained samples were assessed by a flow cytometer (Beckman coulter, Brea, CA) and analyzed by FlowJo software. CD90 used as a marker for a variety of stem cells.

Preparation of endothelial-differentiated AMSCs impregnated alginate beads (vascularized bone construct).

$200 \mathrm{mg}$ of sodium alginate (SA) was dissolved in $10 \mathrm{~mL}$ of PBS, resulting in $2 \%$ sodium alginate solution. The SA solution was heated on a hot plate with stirring thoroughly. The homogeneous SA solution was sterilized using a $0.22 \mu \mathrm{m}$ filter. $10.2 \mathrm{~mL}$ of $1 \mathrm{M} \mathrm{CaCl}_{2}$ stock solution was added to $89.8 \mathrm{~mL}$ of double-distilled water in $100 \mathrm{~mL}$ volumetric flask, resulting in $102 \mathrm{mM} \mathrm{CaCl}_{2}$ solution. The $\mathrm{CaCl}_{2}$ 
solution was sterilized using a $0.22 \mu \mathrm{m}$ filter. The ASCs $\left(5 \times 10^{5} \mathrm{cells} / \mathrm{mL}\right)$ were suspended in SA solution in a $10 \mathrm{~mL}$ conical tube. The mixture of ASCs-SA was dripped into $1 \mathrm{~mL}$ of pre-warmed $\mathrm{CaCl}_{2}$ solution. After incubation at $37^{\circ} \mathrm{C}$ for $5 \mathrm{~min}, \mathrm{CaCl}_{2}$ solution was discarded, and the beads were cultured in 24 well plate with a conventional Endothelial Cell Media 2 (PromoCell, Heidelberg, Germany).

The success of endothelial cell differentiation was confirmed by real-time polymerase chain reaction (PCR) and immunofluorescence staining analysis. For real-time PCR analysis, the samples were retrieved at 3,7 , and 14 days. The total cellular RNA of the cells was extracted with RNeasy Mini Kit (QIAGEN, Hilden, Germany) and reverse-transcribed into cDNA using M-MLV Reverse Transcriptase (Promega, Madison, WI). EZtime Real-Time PCR Premix (Yeastern, Taipei, Taiwan) real-time PCR were used to amplify and simultaneously quantify targeted genes on oryctolagus cuniculus CD31 (Forward: TAAAATCGCCGCAGAGTGGG; Reverse: AGTTCCATTTGATTGGCAGCTC) and Von Willebrand Factor (vWF) (Forward: CCGGCGATGTGTGGACC; Reverse: CCCATGCACATACAGGGACAA). Each Q-PCR was performed in triplicate for PCR yield validation. Data were analyzed by the $2 \triangle \triangle \mathrm{Ct}$ methods, with normalization by the Ct of the housekeeping gene GAPDH (Forward: GGCAAAGTGGATGTTGTCGC; Reverse:

TTCCCGTTCTCAGCCTTGAC). For immunohistochemistry (IHC) staining analysis, the samples were dehydrated and embedded with paraffin after 14 days of induction. These blocks were sectioned with the microtome at $2 \mu \mathrm{m}$ thickness. Sections were stained with hematoxylin and eosin. For antigen retrieval, the specimens were deparaffinized and treated with $0.01 \mathrm{M}$ citrate buffer (Sigma), pH 6.0, in a pressure cooker for $1 \mathrm{~min}$. For blocking of endogenous peroxidase, sections were incubated in $3 \% \mathrm{H}_{2} \mathrm{O}_{2}$ for 10 min. The antibody against platelet endothelial cell adhesion molecule-1 (PECAM-1, CD31) (Bioss, Woburn, MA) and VWF (Bioss) was used at $4^{\circ} \mathrm{C}$ overnight. Then, specimens were incubated in secondary antibody conjugated with Alexa Fluor ${ }^{\circledR} 488$ (Abcam, Cambridge, MA) for 30 min. Hoechst 33342 (Thermo Fisher Scientific) was used to counter staining.

Preparation and scanning electron microscopy (SEM) analysis of engineered periosteum-mimetic cell sheet.

One percent of chitosan solution and collagen solution were prepared using $1 \%$ of acetic acid, respectively and equal volume of them was mixed uniformly. Eight hundred microliter of the mixture was injected into a mold (length: $8.5 \mathrm{~cm}$, width: $3 \mathrm{~cm}$, and height: $0.1 \mathrm{~cm}$ ). The collagen/chitosan membranes were obtained after lyophilization process. The collagen/chitosan membranes were then washed three times at $10 \mathrm{~cm}$ of culture dishes. To make the periosteum-mimetic cell sheet (AMSCs seeded collagen/chitosan membrane), $5 \times 10^{5}$ cells/mL of AMSCs were seeded in the collagen/chitosan membrane evenly at $37^{\circ} \mathrm{C}$ and cultivated with medium composed of low-glucose DMEM medium containing $10 \%$ of $\mathrm{FBS}$ and $1 \%$ of antibiotics for 7 days in a $5 \% \mathrm{CO}_{2}$ incubator. The specimens were dehydrated through a graded series of ethanol solutions, beginning with a $50 \%$ solution and progressing through $70 \%, 95 \%$ and $100 \%$ solutions. The specimens were dried in an HCP-2 critical-point dryer (Hitachi, Tokyo, Japan) and were sputter-coated using an IB.3 ion coater (EiKo, Tokyo, Japan). Finally, the samples were visualized using a field emission SEM (Hitachi S-5000) to determine the matrix formation of AMSCs 
on collagen/chitosan membrane at 7 days. Fluorescence staining was used to observe the distribution of AMSCs in collagen/chitosan membrane.

Preparation of biomimetic vascularized bone-periosteum construct (VBPC).

The endothelial-differentiated AMSCs impregnated alginate beads were washed in a saline buffer first, and then were wrapped with periosteum-mimetic cell sheet to form biomimetic VBPC for further in vivo animal experiment.

In vivo animal experiment.

Twenty-four male New Zealand white rabbits were used and were divided into four groups according to the experimental materials and survived for 12 weeks for final analysis. Figure 1A demonstrated the schematic diagram of the experimental design and groups. Group 1: Acellular alginate beads wrapped with cell sheet (Acellular alginate-sheet construct, $n=6$ ); Group 2: Endothelial-differentiated AMSCs impregnated alginate beads (VBC, $n=6)$; Group 3: Non-differentiated AMSCs impregnated alginate beads wrapped with cell sheet (Non-vascularized AMSCs-alginate-periosteum construct, $n=6$ ); Group 4: Endothelial-differentiated AMSCs impregnated alginate beads wrapped with cell sheet (VBPC, $n=6)$. In order to determine whether the new bone formation and neovascularization were initiated during the early stage or not, one additional rabbit was operated in each experimental group and was sacrificed at 4 weeks. Under anesthesia with an intramuscular injection of Zoletil ( $10 \mathrm{mg} / \mathrm{kgw}$ ) (Virbac Laboratories, Carros, France), the animals underwent intertransverse fusion at the L4-L5 level with different bone graft materials by aseptic manner. The bilateral L4 and L 5 transverse processes were exposed and were decorticated by electric burr. The bone graft material was then placed on each side between the transverse processes (Fig. 1B and C). The fascia and skin were closed layer-by-layer with absorbable sutures. After operation, all animals received $200 \mathrm{mg}$ cefamezine per day for 3 days. The animals were allowed unlimited activity without brace application.

Radiographic examination and bone volume-total volume (BV/TV) analysis.

All the animals underwent 2-mm thin-cut computed tomography (CT) scanning of the lumbosacral spine at 12 weeks. The bone volume (BV) was examined in a defined total volume (TV) by Image J software with the BoneJ plugin. The result of bone volume-total volume ratio (BV/TV) of each group was calculated for new bone formation. Briefly, datasets were rearranged with software, AlignStacks plugin. A computed cylinder (12 mm diameter, $20 \mathrm{~mm}$ height) was set to the region of interest (ROI), which contained the scaffold. As a first step, the grey-value CT data was converted into binaries. The rearranged image sequence was shortened up to the height of $20 \mathrm{~mm}$. Then, the bone volume in this total cylinder (12 $\mathrm{mm}$ diameter, $20 \mathrm{~mm}$ height) was computed with the BoneJ plugin. To gain information about bone growth rates into the different sectors of the scaffold, the total cylinder was subdivided. Horizontal slices of $1 \mathrm{~mm}$ thickness provided information about bone distribution from the surface at the cortical bone to the internal parts of the cylinder, while hollow cylinders around a central core cylinder gave insight into 
bone growth from the periphery to the scaffolds' center. The L4-5 intertransverse fusion areas were collected separately for statistical analysis.

Biomechanical analysis.

The L4-L5 fusion segment was tested for torsional biomechanical strength at weeks 12 post surgery. The L3, L3-4 disc, L5-6 disc, and L6 were embedded along the longitudinal axis in cylindrically shaped epoxy blocks. The length of the L4-L5 non-embedded portion of each specimen was kept identical. The potted samples were then mounted on a Material Testing System machine (GT-7054-A1) (GOTECH Testing Machines Inc., Taichung, Taiwan) for rotational torque assessments. Each individual specimen was tested until ultimate failure in external rotation along its longitudinal axes at 1 degree per second. The maximum torque values were obtained from the torque-rotation angle curve. The results of maximum torque value were presented as means \pm standard deviation (SD).

Histology evaluation.

The enbloc spine specimens were fixed in $10 \%$ neutral buffered formaldehyde, decalcified, dehydrated through alcohol gradients, cleared and embedded in paraffin blocks. Tissue blocks of the intertransverse fusion areas were sectioned and stained with hematoxylin and eosin (H\&E) and Masson's trichrome methods, and visualized using standard light microscopy. For immunohistochemistry staining, the slide firstly was blocked with normal goat serum for $45 \mathrm{~min}$. Mouse anti-rabbit monoclonal primary antibodies of CD 31 diluted 1:100 (Novus Biologicals, Littleton, CO) were applied at $4^{\circ} \mathrm{C}$ for $24 \mathrm{~h}$, followed by incubation with HRP-conjugated anti-mouse secondary antibody diluted 1:250. Diaminobenzidine (Sigma-Aldrich) was used as the substrate to develop brown color in the presence of CD31. The slides were dehydrated before being cover slipped.

\section{Statistical analysis}

Statistical analyses were performed using the Statistical Package for the Social Sciences for Windows (SPSS, version 12.0; IBM, Armonk, NY, USA). Numerical data are expressed as mean value \pm standard deviation (SD). Statistical analysis was performed by analysis of variance (ANOVA) to determine statistical significance. For the histomorphometry data and mechanical strength comparison between groups, the maximal values were analyzed using two-tailed Student's test. A statistical significant difference was set at $p<0.05$.

\section{Results}

Identification of rabbit adipose-derived stem cells (AMSCs).

Cell morphologies of non-differentiated AMSCs presented a spindle shape for three passages and were spreading evenly on the culture dish (Fig. 2A). Furthermore, the surface markers of AMSCs stained with surface antigens associated with mesenchymal stem cells (CD9, CD29, CD44, CD73, CD90 and CD105) 
and surface antigens associated with endothelium and hematopoiesis (CD45 and CD34) were characterized. Flow cytometry results revealed that AMSCs expressed CD9 (89.95\%), CD29 (91.27\%), CD44 (68.04\%), CD73 (72.40\%), CD90 (84.52\%) and CD105 (85.61\%). However, surface markers such as CD45 and CD34 associated with endothelium and hematopoiesis were not expressed (Fig. 2B).

Endothelial differentiation of seeded AMSCs in alginate beads..

The mRNA expressions of two factors, PECAM-1(CD31) and vWF, were conducted to observe the vascularization of epithelial-differentiated AMSCs impregnated alginate beads as well as the immunofluorescence staining. The PECAM-1 mRNA expressions of endothelial-differentiated AMSCs showed 2.3, 2.6 and 14.3-fold higher after 3-day, 7-day and 14-day cultivation compared to nondifferentiated AMSCs. For vWF mRNA expression, the endothelial-differentiated AMSCs also showed 17.5-fold higher after 14-day cultivation compared to non-differentiated AMSCs. The mRNA expressions of PECAM-1 and vWF showed markedly up regulation after endothelial differentiation and increased sequentially by cultivation duration (Fig. $3 A$ and B). Notably, further time course analyses of mRNA expression showed a sharply increase of mRNA expression presented at days 14 cultivation, indicating a key time point for AMSCs to obtain the essential endothelial phenotype when cultured in alginate scaffolds. After 14 days induction, the H\&E stained histology of epithelial-differentiated AMSCs impregnated alginate beads showed the cells were evenly distributed within the porous alginate scaffold (Fig. 3C). The success of endothelial cell differentiation of AMSCs was confirmed by immunofluorescent staining of PECAM-1 and VWF (Fig. 3D and E). In addition, self-organized tube-like structures were found (Fig. 3E). It indicated that the vascularized alginate beads had the capability of angiogenesis for further vascular network formation in vitro.

Analysis of periosteum-mimetic cell sheet.

The gross view and size of collagen/chitosan cell sheet is $8.5 \mathrm{~cm}$ of length, $3 \mathrm{~cm}$ of width and $0.1 \mathrm{~cm}$ of height (Fig. 3F). The periosteum-mimetic cell sheets were obtained when AMSCs were cultivated on collagen/chitosan cell sheet for seven days. The morphology and structures were analyzed using SEM. The morphology of the biomimetic cell sheet showed an irregular lamina structures with interconnected pores. The diameter of the interconnected pore was around $50 \mu \mathrm{m}$ (Fig. 3G) and might be a suitable geometry for implanted cell growth. The AMSCs grew in a flat form from the surface to interior of the cell sheet. Confocal microscopic results further proved that most AMSCs grew with a thin layer form on the surface of cell sheet and then ingrown into the interior area via interconnected pores (Fig. $3 \mathrm{H}$ ). These results indicated that AMSCs could adhere to the cell sheet for further survival, proliferation, and differentiation.

Radiographic results of in vivo animal experiment.

The results of new bone formation and ossification at L4-5 were determined by 3D CT scan reconstructive images. Figure 4 illustrated the degree of new bone formation and ossification among study groups at 12 weeks after surgery. The reconstructive coronary, axial, and sagittal cut images 
showed bone formation between the L4 and L5 transverse processes in all four groups, but the degree of bony ossification and continuity was different. In Group 1, reconstructive CT images showed continuous bone mass formation between L4 and L5 transverse processes. The ossified area only located at the outer region (periosteum-mimetic cell sheets), while the inner region (acellular alginate beads) was not ossified (Fig. 4A1-3). Unlike the other three study groups (implanted grafts with cell sheet wrapping), reconstructive CT images from Group 2 showed interrupted and paper-thin bone formation between the bilateral L4 and L5 transverse processes although highly ossification was noted. The new bone mass became flat pattern might be caused by the force of overlaying muscle compresses to the implanted alginate beads owing to lack of cell-sheet support (Fig. 4B1-3). In Group 3, continuous bone mass formation between transverse processes with ossification at the outer region (periosteum-mimetic cell sheets) was noted, while scattered ossification spots were observed in the center area (non-vascularized alginate beads) (Fig. 4C1-3). In Group 4, continuous bone mass formation with evenly distributed ossification at central and outer regions between L4-5 transverse processes was noted (Fig. 4D1-3). The reconstructive 3D CT images revealed a uninterrupted and bridging bone mass between bilateral L4 and L5 transverse processes in Group 1 (Fig. 4A4), Group 3 (Fig. 4C4), and Group 4 (Fig. 4D4). However, Group 2 could not form a continuously bone structure (Fig. 4B4).

For quantitative evaluation of matured bone formation and ossification, the result of bone volume-total volume (BV/TV) ratio was showed in Fig. 4E. The BV/TV ratio of each group was as follows: $0.67 \%$ for Group 1; $0.73 \%$ for Group 2; $1.21 \%$ for Group 3; and 2.28\% for the Group 4. Group 4 had 3.4-fold, 3.1-fold, and 1.9-fold more bone volume than Group $1(p<0.01)$, Group $2(p<0.01)$, and Group $3(p=0.65)$. There was a tendency towards a higher volume of regenerated bone in Group 4. These results indicated that VBPC facilitated to the new bone formation and maturation in vivo.

Biomechanical strength evaluation.

At weeks 12 post-surgery, L4-5 fusion segments were secured onto mechanical strength machine for maximal rotational torque evaluation. The average maximal torques at failure of Group 1, Grou2, Group 3 , and Group 4 were $367.17 \pm 38.59 \mathrm{~N}-\mathrm{mm}, 306.49 \pm 74.25 \mathrm{~N}-\mathrm{mm}, 331.31 \pm 45.77 \mathrm{~N}-\mathrm{mm}$, and $372.69 \pm 27.97$ $\mathrm{N}$-mm respectively (Fig. 4F). The maximal torque mean value for Group 4 was higher than the other groups, but there was not statistically significant $(p=0.085)$. Of note, the maximal torque was significantly lower for Group 2 (alginate beads without cell sheet wrapping) than the other three study groups (alginate beads with cell sheet wrapping), suggesting the cell sheet likely played a critical role for mechanical support of alginate beads. Besides, the maximal torque for Group 4 was marginally higher than Group $3(p=0.055)$, suggesting superior bone formation and ossification those were likely due to the capacity for neovascularization of VBPC graft.

Histology evaluation.

Histological analyses for healing processes and new bone formation for the four study groups were performed with consecutive sections by routine H\&E and Masson's trichrome staining at 4 weeks and 12 weeks. The transverse processes (tp, host bone), newly formed bone (nb), collagen/chitosan sheet (s), 
and residual alginate beads $(\mathrm{g})$ are highlighted in Fig. 5. Histological sections at 4 week were examined to determine whether bone and capillary formation was initiated during the early stage or not. Overview of the healing processes and responses was presented at the 12 week end point. No new bone or microcapillary-like structure formation was observed in all four groups at 4 weeks. There was no evidence of inflammatory response to the artificial grafts in any specimens since no lymphocytic cells were detected. Notably, except cell-free Group 1, obvious cellular micro-pores within the alginate beads were found in other three cell-seeded study groups, which indicated the impregnated cells survived and grew within the alginate beads during the early stage (Fig. 5A1-4 and B1-4). After 12 weeks of survival, the alginate beads were degraded into tiny remnants and scattered within the engineered composites in all groups. There were persistent cellular micro-pores within the alginate remnants in Group 2 and Group 4 (Fig. 5C2\&4 and D2\&4). Self-organized tube-like structures could be observed within and between the vascularized alginate remnants, indicative of new microvascular formation via angiogenesis by the impregnated endothelial-differentiated AMSCs. On the contrary, no pores or cells could be observed within the alginate remnants for Group 1 and Group 3 (Fig. 5C1\&3 and D1\&3). It indicated that without endothelial differentiation, AMSCs could not exist within alginate beads till late stage for further new bone formation.

At 12 weeks, different degree of new bone formation within the intertransverse fusion areas was observed in all groups. Thin and scattered trabecular bone formation without bridging between alginates could be observed for Group 1 (Fig. 5C1 and D1). Thick and bridged connecting trabecular bone formation was visible between alginate scaffolds for Group 2 and Group 4 (Fig. 5C2\&4 and D2\&4). In Group 3 specimens, thin and scattered bone formation near the transverse process and amount of fibrous tissues between alginate scaffolds could be observed (Fig. 5C3-4). In addition, to examine the neovascularization of bone graft in vivo, the immunohistochemistry stain of $\mathrm{CD} 31^{+}$blood vessel formation in fusion area was carried out and semi-quantitative analysis of the brown area was counted. The $\mathrm{IHC}$ results indicated significantly better performance of $\mathrm{CD} 31^{+}$blood vessel formation appeared for Group 2 (Fig. 6B) and Group 4 (Fig. 6D) than those for Group 1 (Fig. 6A) and Group 3 (Fig. 6C). Semiquantitative analysis of the $\mathrm{CD} 31^{+}$immunohistochemistry stained brown area confirmed that the newly formed blood vessel density for Group 2 and Group 4 were higher than that for Group 1 and Group 3 owing to the endothelial differentiation of AMSCs (Fig. 6E). These results indicated that the capacity for angiogenesis of epithelial-differentiated AMSCs impregnated alginate beads in vivo.

\section{Discussion}

Successful reconstruction of skeletal defects often requires bone grafting for repairing. Although the autogenous bone grafting remains the gold standard, several concerns such as limited availability, donor site morbidities, increased blood loss, and increased operative time have prompted the search for feasible alternatives [1-4]. In recent years, many types of biomaterials such as bone marrow, demineralized bone matrix, collagen, and ceramics have been created for bone graft surgery to solve the defects of autogenous bone grafts [20]. However, the current artificial bone grafts have failed to achieve anticipated 
osteogenesis owing to their insufficient neovascularization capacity [4]. To overcome the deficit, the authors aimed to develop a cell-based biomimetic vascularized periosteum-bone bone graft to create a superior neovascularization and nutritional environment for further cell growth and osteogenesis. The result was promising and showed that incorporation of epithelial-differentiated AMSCs impregnated alginate beads and periosteum-mimetic cell sheet could create a biomimetic vascularized periosteumbone construct and facilitate new bone formation as assessed in a rabbit spine fusion model. These findings implicate a relatively new approach to improve the difficult bone defect management.

Recently, AMSCs are paid attractive by the researchers due to the ease of obtaining these cells from small amounts of fat tissues or via liposuction versus painful bone marrow aspirates [21]. Additionally, the AMSCs has been proved with a higher rate of calcium deposition and a higher rate of proliferation than the bone marrow mesenchymal stem cells in an ex vivo calvarial defects of a rabbit model [22]. Additionally, AMSCs from subcutaneous fat tissue has been proved with the potential to secret multiple synergistic proangiogenic growth factors [8]. These evidences explained the merits of using AMSCs as a feasible cell source in bone defect repairing surgery.

Prevascularization is a prospective strategy to facilitate the vascularization of an implanted scaffold in vivo, demonstrating the quick integration of functional vascular networks with the host vascular system $[23,24]$. However, there is a challenge to get effective induction of blood vessels into engineered graft in tissue engineering. The major obstacle is to obtain an abundant source of efficient autologous endothelial cells [25]. Reports proved that MSCs have the multipotent ability to differentiate into osteoblast and endothelial cells $[26,27]$. Therefore, AMSCs was induced and differentiated into endothelial-like cells by VEGF to solve the problem of the cell source of endothelial cells. Pankajakshan et al. successfully induced porcine mesenchymal stem cells to endothelial cells, which provided new options for re-endothelialization [28]. Liu et al. demonstrated that the co-culture rabbit MSC-derived endothelial cells improved the osteogenesis of MSCs and promoted new bone formation [29]. Our experimental results ex vivo indicated that the AMSCs cultivated into alginate beads had the ability to differentiate into endothelial-like cells via the mRNA analysis of PECAM-1 and vWF. The self-organized tube-like structures within the scaffold appeared which indicated formation of blood vessels after endothelial differentiation. Furthermore, our in vivo results also agreed with this phenomenon, indicating that using the vascularized bone-periosteum composite could induce neovascularization around the implanted area to promote further new bone formation and ossification.

Periosteum is often used as a cell source for bone tissue engineering due to possessing a population of stem cells and osteoprogenitors. Similar to commercial bone grafts, development of engineeredmembrane technology is used to replace periosteum owing to obstacles for obtaining periosteum at the traumatic site [30]. Several engineered-membrane technologies using collagen gel scaffold, small intestinal submucosa matrix, or thrombin-fibrinogen membrane have been reported recently [31-33]. These membranes are basically composed of collagen. Their results showed these artificial periosteums were particularly beneficial for bone regeneration. Additionally, the chitosan arises as one of the prominent materials due to its inherent biocompatibility, antibacterial, hemostatic, and healing properties 
[34]. Kung et al. utilized chitosan-collagen composites with in vivo osteoinductive effect to wrap around pure titanium implant surfaces. Their results showed chitosan-collagen composites might induce in vivo new bone formation around pure titanium implant surfaces [35]. Li et al. proved chitosan-collagen membranes can enhance bone regeneration in dog dehiscence-type defect model and could be a candidate for use in guided bone regeneration [36]. Gao et al. demonstrated nanofiber chitosan-collagen membrane can promote new bone regeneration effectively on guided bone regeneration [37]. These reports indicated the combination of collagen and chitosan as a membrane is considered as a promising periosteum substitute for bone regeneration. Besides, the cell sheet might play an important role for preventing the alginate beads from early absorption and squeezing effect by the surrounding host tissues. As the illustrated results, the newly formed bone became flatten and discontinuous owing to lack of cell sheet support as the radiographic findings in Group 2. On the contrary, results of Group 3 and Group 4 showed that the cell sheet not only provided the benefit for osteogenesis but also provided the mechanical support for alginate scaffolds for further bone regeneration.

One critical point for creating a tissue engineering biomimetic bone graft is to successfully integrate the prevascularized scaffolds with the artificial periosteum into a membrane/scaffold complex. Two vital factors, cell-cell interaction and properties of scaffolds, deeply affect the success of new bone formation using an artificial biomimetic periosteum-bone construct. A report explained tissue ingrowth might be limited because of intrinsic geometrical and structural characteristics of 3D scaffolds [38]. The current results showed that the vascularized periosteum-bone construct has better neovascularization and bone formation when compared to non-vascularized grafts. In the current study, we fabricate a porous cellular membrane seeded with AMSCs to mimic native periosteum. We then combined with alginate scaffolds to produce a biomimetic periosteum-bone construct for application in the regeneration of spinal fusion surgery. The histological results in vitro revealed AMSCs could grow evenly within the porous scaffolds via the interconnected micropores. Additionally, these interconnected micropores allow the circulating of nutrients and growth factors within the whole biomimetic construct, promoting a synergistic effect in between the new blood vessel production, new bone formation and mineralization.

Another critical point to affect the new bone formation in vivo is considered for cells, i.e. undifferentiated AMSCs, differentiated AMSCs (endothelial-like cells) and host cells. Reports showed driving stem cell differentiation toward the needed lineages has been studied increasingly via co-culture of MSCs with other mature cells [5-8]. The cell behaviors occurred and changed either by direct or indirect cell-cell contacts between the cell types. The mechanism of these cells interacted each other remain unclear. The microenvironment and extracellular matrix (ECM) formed by cells may play a crucial role in osteogenic differentiation [39]. The ECM produced from these cells provides an appropriate microenvironment to support cell adhesion and direct cell behaviors, such as proliferation and differentiation [40, 41]. The current results showed that the numbers of newly formed blood vessels and areas of bone formation were higher in the specimens of Group 4 than that of the other groups. It might be owing to the endothelial differentiation of AMSCs for further angionesis, which indicate more nutrients and growth factors could be obtained in the vascularized periosteum-bone groups. Although the current study does not identify the real impact factors involved in the in vivo situation for new bone formation in detail, the 
results demonstrate the possibility of appropriate growth factors production in the microenvironment from the interaction of undifferentiated AMSCs, differentiated AMSCs (endothelial-like cells) and host

cells. Currently, issues such as the detailed mechanism of cytokine secretion, triggered by cell-cell or cellscaffold interactions remain to be clarified. In this vein, we will continue our endeavors to elucidate the mechanisms of MSC-facilitated bone regeneration in the context of tissue engineering.

\section{Conclusions}

This study demonstrated that the novel tissue engineered biomimetic vascularized bone-periosteum construct can promote neovascularization and bone regeneration in a rabbit spine fusion model in vivo. The successful differentiation of AMSCs into endothelial-like cells within alginate scaffold provides the endothelial cell source for angiogenesis. The collagen/chitosan cell sheet not only acts as a biomimetic periosteum but also offer endothelial-differentiated AMSCs impregnated alginate beads a mechanical support for further bone regeneration. This strategy demonstrates promising potential for future bone tissue engineering to regenerate bone defects and to enhance spinal fusion success. It may provide a novel approach to create a superior blood supply and nutritional environment for osteogenesis and to overcome the deficits of insufficient vascularization capacity of current artificial bone graft substitutes.

\section{Abbreviations}

VBPC: Vascularized bone-periosteum construct; AMSCs: Adipose-derived mesenchymal stem cells; VBC: Vascularized bone construct; MSCs: Mesenchymal stem cells; FGF-2: Fibroblast growth factor-2; VEGF: Vascular endothelial growth factor; 3D: Three-dimension; PBS: Phosphate-buffered saline; FBS: Fetal bovine serum; DMEM: Dulbecco's modified Eagle's medium; EDTA: Ethylene diamine tetraacetic acid; FITC: Fluorescein isothiocyanate; CD: Cluster of differentiation; SA: Sodium alginate; PCR: Polymerase chain reaction; IHC: Immunohistochemistry; vWF: Von Willebrand Factor; PECAM-1: Platelet endothelial cell adhesion molecule-1; SEM: Scanning electron microscopy; CT: Computed tomography; BV/TV: Bone volume-total volume ratio; ROI: Region of interest; H\&E: Hematoxylin and eosin; SD: Standard deviation; ANOVA: Analysis of variance; ECM: Extracellular matrix

\section{Declarations}

\section{Ethics approval and consent to participate}

All experimental procedures and the use of research animals in this study were approved by the Institutional Animal Care and Use Committee (IACUC) at the authors' institute (Affidavit of Approval of Animal Use Protocol Chang Gung Memorial Hospital permit number: 2015121201 )

\section{Consent for publication}

Not applicable 
Availability of data and materials

The datasets generated and/or analyzed during the current study are not publicly available but are available from the corresponding author upon reasonable request.

\section{Competing interests}

The authors have no competing interests to declare.

\section{Funding}

This work was supported by authors' institute "Chang Gung Memorial Hospital, Keelung (Grant numbers: CMRPG2G0201, CMRPG2I0051 and CMRPG2H0071)".

\section{Authors' contributions}

$T F, Y W, C C$, and TL contributed to the study conception, study design, and study performance. WC and CW contributed to data collection, data analysis and interpretation. TF and WC performed the preparation and revision of the manuscript. All authors read and approved the final article.

\section{Acknowledgements}

The authors gratefully acknowledge the financial support provided by Chang Gung Memorial Hospital, Keelung (CMRPG2G0201, CMRPG2I0051 and CMRPG2H0071).

\section{References}

1. Gumbs AA, Bloom ND, Bitan FD, Hanan SH. Open anterior approaches for lumbar spine procedures. Am J Surg. 2007;194:98-102.

2. Vaccaro AR, Chiba K, Heller JG, Patel TC, Thalgott JS, Truumees E, et al. Bone grafting alternatives in spinal surgery. Spine J. 2002;2:206-15.

3. Fernyhough JC, Schimandle JJ, Weigel MC, Edwards CC, Levine AM. Chronic donor site pain complicating bone graft harvesting from the posterior iliac crest for spinal fusion. Spine. 1992;17:1474-80.

4. Boden SD. Biology of lumbar spine fusion and use of bone graft substitutes: present, future, and next generation. Tissue Eng. 2000;6:383-99.

5. Khaled EG, Saleh M, Hindocha S, Griffin M, Khan WS. Tissue engineering for bone production- stem cells, gene therapy and scaffolds. Open Orthop J. 2011;5(Suppl 2):289-95.

6. Cha C, Liechty WB, Khademhosseini A, Peppas NA. Designing biomaterials to direct stem cell fate. ACS Nano. 2012;6:9353-8.

7. Zhang R, Ma J, Han J, Zhang W, Ma J. Mesenchymal stem cell related therapies for cartilage lesions and osteoarthritis. Am J TransI Res. 2019;11:6275-89. 
8. Rehman J, Traktuev D, Li J, Merfeld-Clauss S, Temm-Grove CJ, Bovenkerk JE, et al. Secretion of angiogenic and antiapoptotic factors by human adipose stromal cells. Circulation. 2004;109:12928.

9. Sharma A, Brand D, Fairbank J, Ye H, Lavy C, Czernuszka J. A self-organising biomimetic collagen/nano-hydroxyapatite-glycosaminoglycan scaffold for spinal fusion. J Mater Sci. 2017;52:12574-92.

10. Vater CA, Harris ED, Siegel RC. Native cross-links in collagen fibrils induce resistant to human synovial collagenase. Biochem J. 1979;181:639-45.

11. Ghaemi SR, Delalat B, Cetó X, Harding FJ, Tuke J, Voelcker NH. Synergistic influence of collagen I and BMP 2 drives osteogenic differentiation of mesenchymal stem cells: A cell microarray analysis. Acta Biomater. 2016;34:41-52.

12. Ren L, Kang Y, Browne C, Bishop J, Yang Y. Fabrication, vascularization and osteogenic properties of a novel synthetic biomimetic induced membrane for the treatment of large bone defects. Bone. 2014;64:173-82.

13. Chen J, Zhang D, Li Q, Yang D, Fan Z, Ma D, et al. Effect of different cell sheet ECM microenvironment on the formation of vascular network. Tissue Cell. 2016;48:442-51.

14. Elgali I, Turri A, Xia W, Norlindh B, Johansson A, Dahlin C, et al. Guided bone regeneration using resorbable membrane and different bone substitutes: Early histological and molecular events. Acta Biomater. 2016;29:409-23.

15. Neves SC, Teixeira LSM, Moroni L, Reis RL, Blitterswijk CAV, Alves NM, et al. Chitosan/poly(epsiloncaprolactone) blend scaffolds for cartilage repair. Biomaterials. 2011;32:1068-79.

16. Nettles DL, Elder SH, Gilbert JA. Potential use of chitosan as a cell scaffold material for cartilage tissue engineering. Tissue Eng. 2002;8:1009-16.

17. Sellimi S, Younes I, Ayed HB, Maalej H, Montero V, Rinaudo M, et al. Structural, physicochemical and antioxidant properties of sodium alginate isolated from a Tunisian brown seaweed. Int $\mathrm{J}$ Biol Macromol. 2015;72:1358-67.

18. Nguyen VT, Ko SC, Oh GW, Heo SY, Jeon YJ, Park WS, et al. Anti-inflammatory effects of sodium alginate/gelatine porous scaffolds merged with fucoidan in murine microglial BV2 cells. Int J Biol Macromol. 2016;93:1620-32.

19. Tiwari S, Patil R, Bahadur P. Polysaccharide based scaffolds for soft tissue engineering applications. Polymers (Basel). 2018;11:1.

20. Lobb DC, DeGeorge BR Jr, Chhabra AB. Bone graft substitutes: current concepts and future expectations. J Hand Surg Am. 2019;44:497-505.

21. Storti G, Scioli MG, Kim BS, Orlandi A, Cervelli V. Adipose-derived stem cells in bone tissue engineering: useful tools with new applications. Stem Cells Int. 2019;Nov 6:3673857.

22. Lin L, Shen Q, Wei X, Hou Y, Xue T, Fu X, et al. Comparison of osteogenic potentials of BMP4 transduced stem cells from autologous bone marrow and fat tissue in a rabbit model of calvarial defects. Calcif Tissue Int. 2009;85:55-65. 
23. Sakai Y, Nishikawa M, Evenou F, Hamon M, Huang H, Montagne KP, et al. Engineering of implantable liver tissues. Methods Mol Biol. 2012;826:189-216.

24. Johnson EO, Troupis T, Soucacos PN. Tissue-engineered vascularized bone grafts: basic science and clinical relevance to trauma and reconstructive microsurgery. Microsurgery. 2011;31:176-82.

25. Zhou L, Niu X, Liang J, Li J, Li J, Cheng Y, et al. Efficient differentiation of vascular endothelial cells from dermal-derived mesenchymal stem cells induced by endothelial cell lines conditioned medium. Acta Histochem. 2018;120:734-40.

26. Böhrnsen F, Schliephake H. Supportive angiogenic and osteogenic differentiation of mesenchymal stromal cells and endothelial cells in monolayer and co-cultures. Int J Oral Sci. 2016;8:223-30.

27. Ren L, Ma D, Liu B, Li J, Chen J, Yang D, et al. Preparation of three-dimensional vascularized MSC cell sheet constructs for tissue regeneration. Biomed Res Int. 2014;Jul 8:301279.

28. Pankajakshan D, Kansal V, Agrawal DK. In vitro differentiation of bone marrow derived porcine mesenchymal stem cells to endothelial cells. J Tissue Eng Regen Med. 2013;7:911-20.

29. Liu J, Liu C, Sun B, Shi C, Qiao C, Ke X, et al. Differentiation of rabbit bone mesenchymal stem cells into endothelial cells in vitro and promotion of defective bone regeneration in vivo. Cell Biochem Biophys. 2014;68:479-87.

30. Li N, Song J, Zhu G, Li X, Liu L, Shi X, et al. Periosteum tissue engineering- a review. Biomater Sci. 2016;4:1554-61.

31. Gellynck K, Shah R, Deng D, Parkar M, Liu W, Knowles JC, et al. Cell cytoskeletal changes effected by static compressive stress lead to changes in the contractile properties of tissue regenerative collagen membranes. Eur Cell Mater. 2013;25:317-25.

32. Lindberg K, Badylak SF. Porcine small intestinal submucosa (SIS): a bioscaffold supporting in vitro primary human epidermal cell differentiation and synthesis of basement membrane proteins. Burns. 2001;27:254-66.

33. Göbel K, Eichler S, Wiendl H, Chavakis T, Kleinschnitz C, Meuth SG. The coagulation factors fibrinogen, thrombin, and factor XII in inflammatory disorders- a systematic review. Front Immunol. 2018;9:1731.

34. Rajoka MSR, Zhao L, Mehwish HM, Wu Y, Mahmood S. Chitosan and its derivatives: synthesis, biotechnological applications, and future challenges. Appl Microbiol Biotechnol. 2019;103:1557-71.

35. Kung S, Devlin H, Fu E, Ho KY, Liang SY, Hsieh YD. The osteoinductive effect of chitosan-collagen composites around pure titanium implant surfaces in rats. J Periodontal Res. 2011;46:126-33.

36. Li X, Wang X, Zhao T, Gao B, Miao Y, Zhang D, et al. Guided bone regeneration using chitosancollagen membranes in dog dehiscence-type defect model. J Oral Maxillofac Surg. 2014;72:304e114.

37. Gao B, Li XJ, Lin M, Li YY, Dong Y. Development of a novel absorbable nanofiber chitosan-collagen membrane by electrospinning and the preliminary study on guided bone regeneration. Zhonghua Kou Qiang Yi Xue Za Zhi. 2018;53:85-91. 
38. Zhang D, Gao P, Li Q, Li J, Li X, Liu X, et al. Engineering biomimetic periosteum with $\beta$-TCP scaffolds to promote bone formation in calvarial defects of rats. Stem Cell Res Ther. 2017;8:134.

39. Fu TS, Wang YC, Chen $\mathrm{CH}$, Chang CW, Lin TY, Wong CB, et al. Engineered periosteum-bone biomimetic bone graft enhances posterolateral spine fusion in a rabbit model. Spine J. 2019;19:762-71.

40. Badylak SF, Freytes DO, Gilbert TW. Extracellular matrix as a biological scaffold material: Structure and function. Acta Biomater. 2009;5:1-13.

41. Reilly GC, Engler AJ. Intrinsic extracellular matrix properties regulate stem cell differentiation. $J$ Biomech. 2010;43:55-62.

\section{Figures}
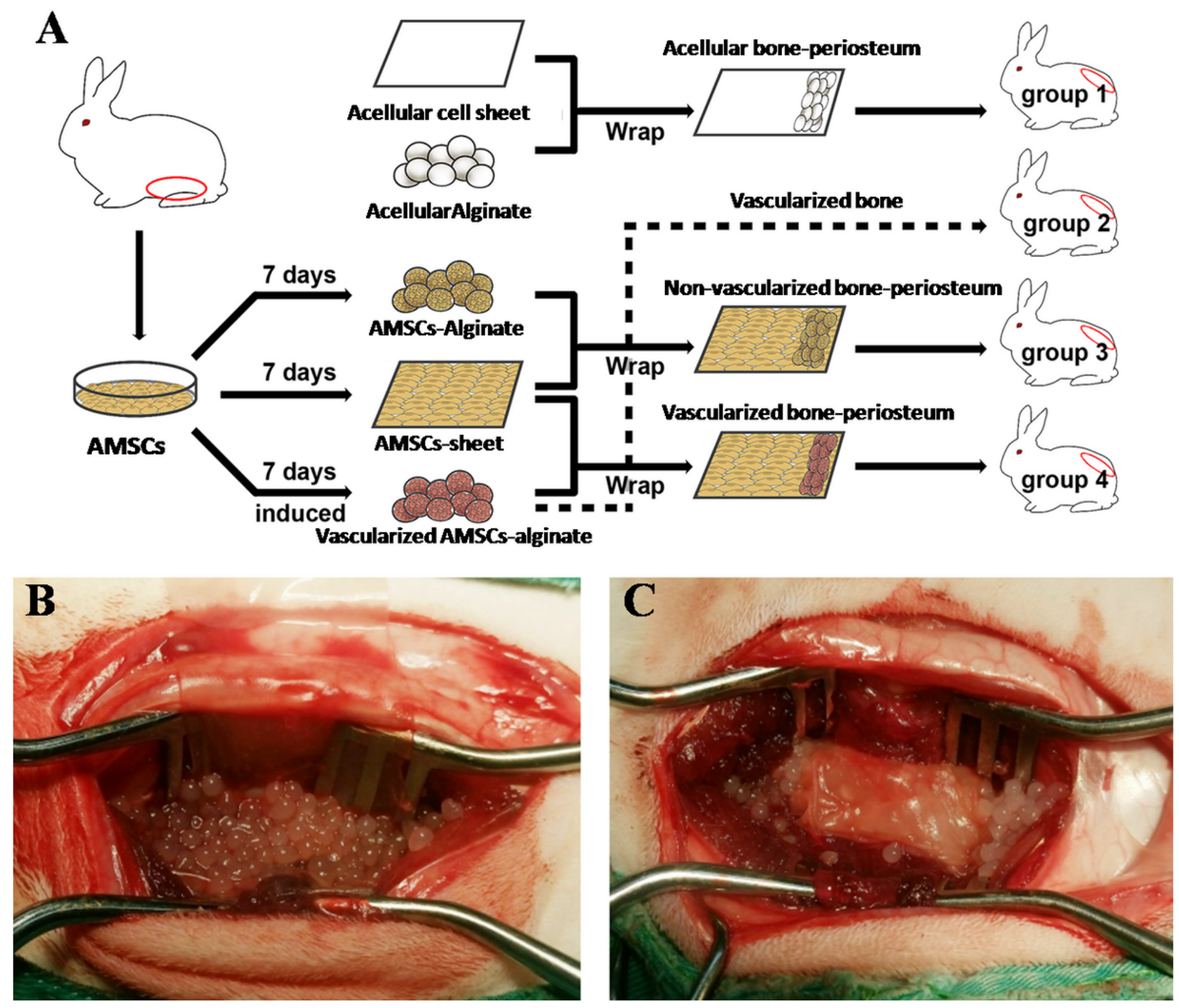


\section{Figure 1}

Schematic diagram of the experimental designs and bone graft materials. (a) representative the four study groups and whole procedures of the experiment. The alginate beads were wrapped with collagen/chitosan cell sheet to fabricate a bone-periosteum composite for further in vivo experiment. Gross appearance of the alginate beads (b) and biomimetic bone-periosteum composite (c) implanted between the $L 4$ and $L 5$ transverse processes of a rabbit posterolateral spinal fusion model.
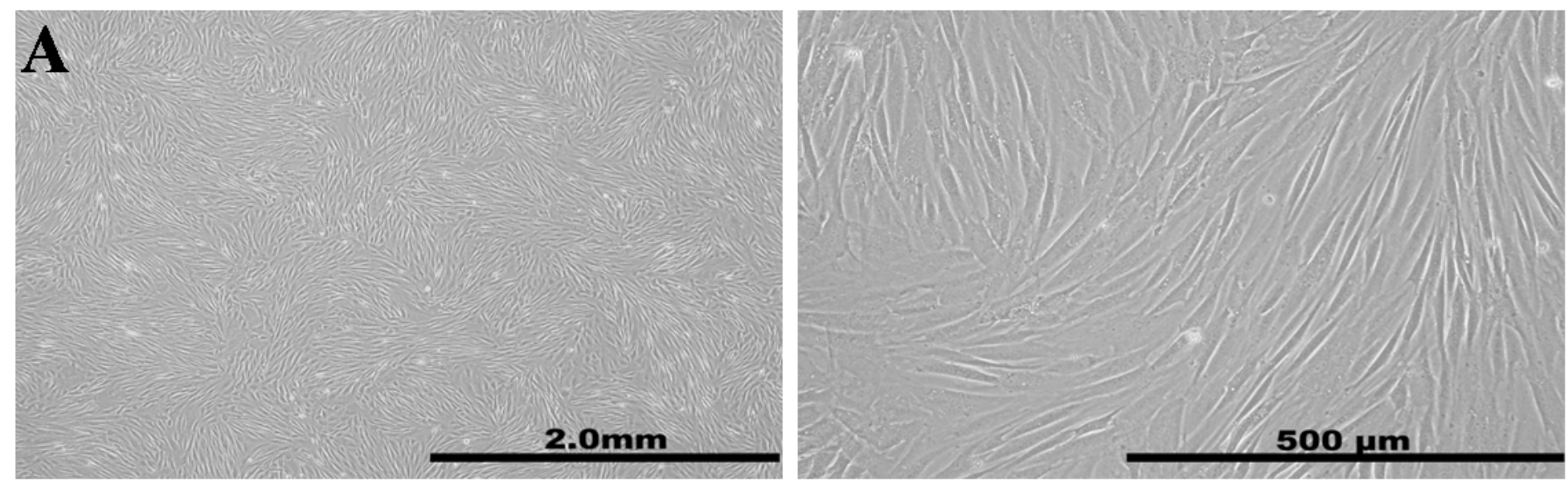

B
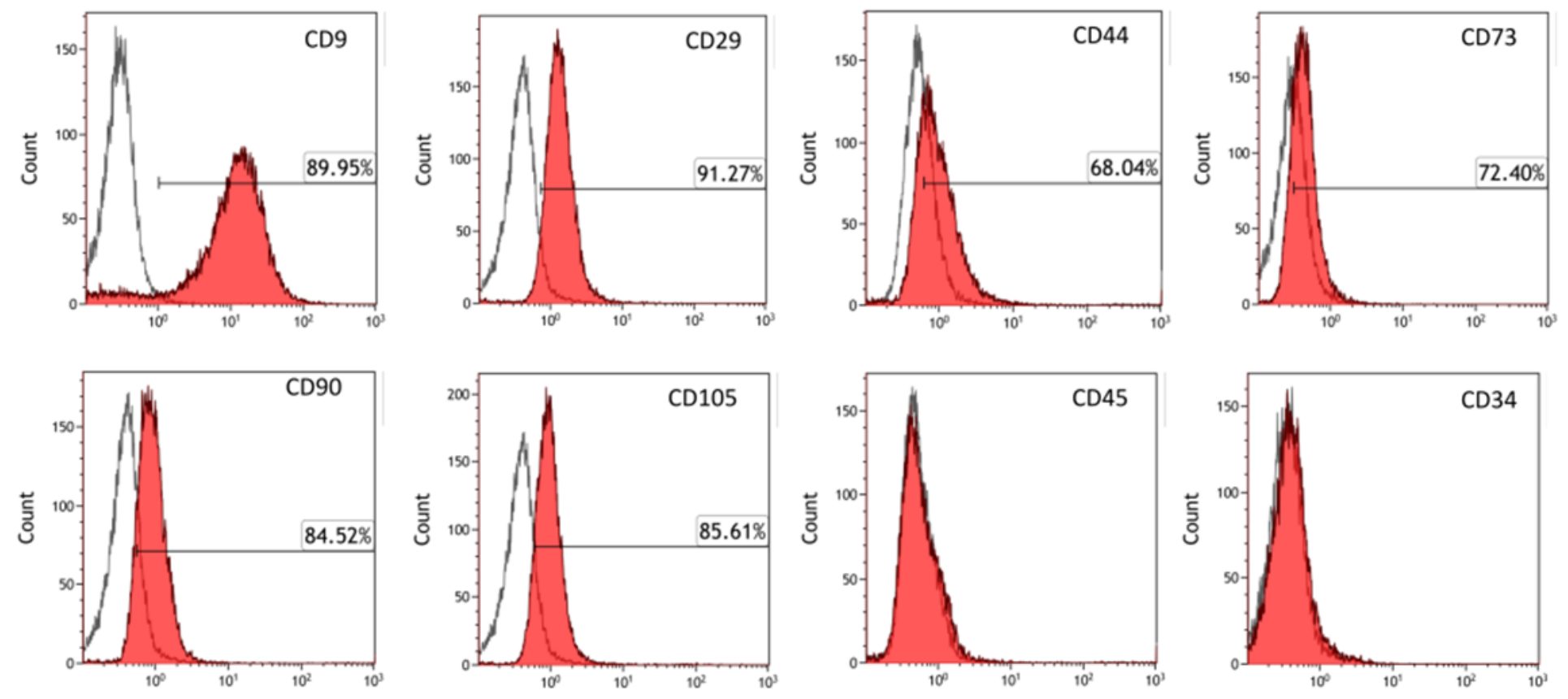

\section{Figure 2}

Cell morphologies and the surface markers of AMSCs. (a) representative the low and high magnification of passage three non-differentiated AMSCs morphologies and showed spindle shape spreading evenly on the culture dish. (b) representative data from flow cytometry results showed expression of CD9 (89.95\%), CD29 (91.27\%), CD44 (68.04\%), CD73 (72.40\%), CD90 (84.52\%) and CD105 (85.61\%) surface antigens 
associated with mesenchymal stem cells. However, CD45 and CD34 surface markers associated with endothelium and hematopoiesis were not expressed.
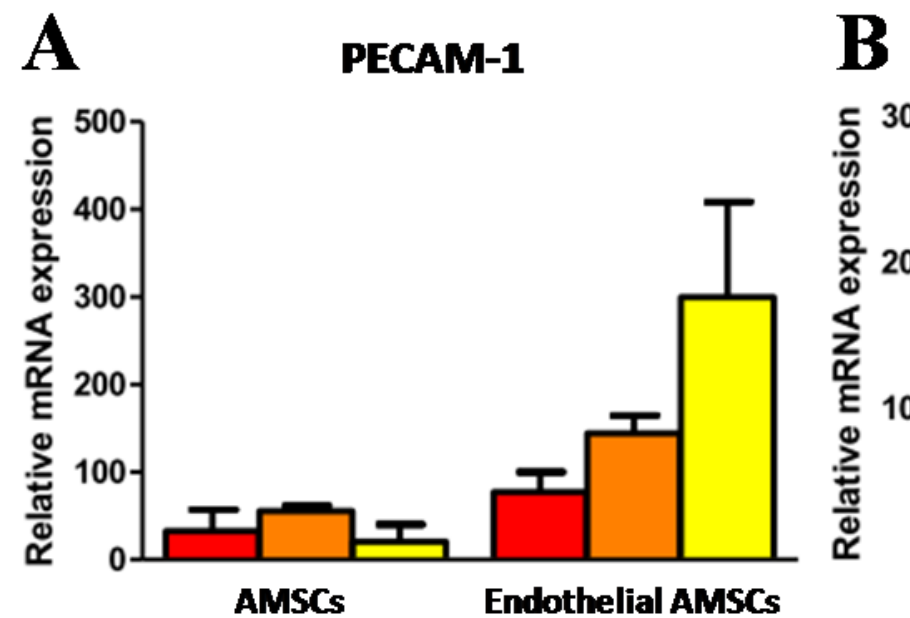

vWF
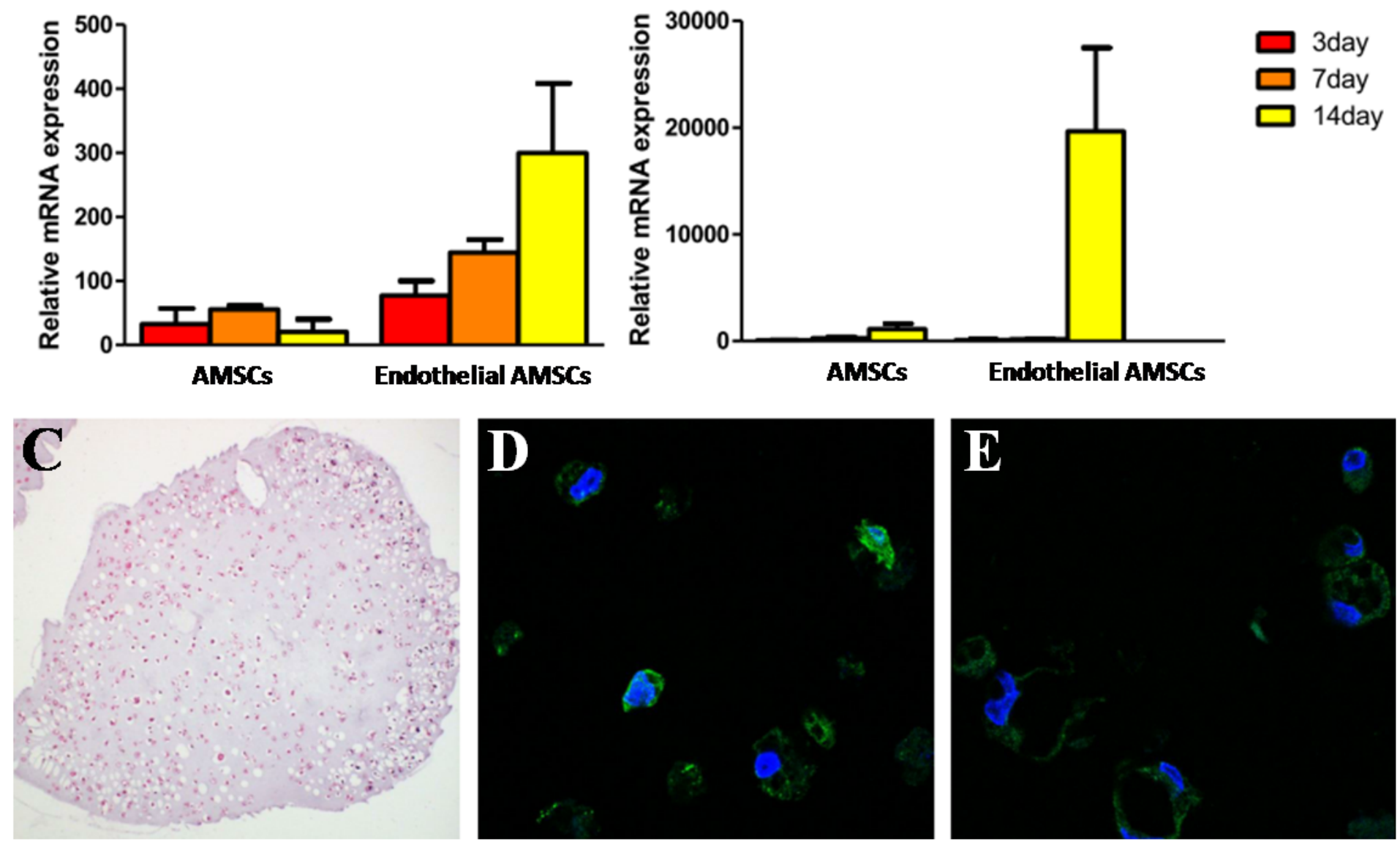

\section{$\mathbf{F}$}
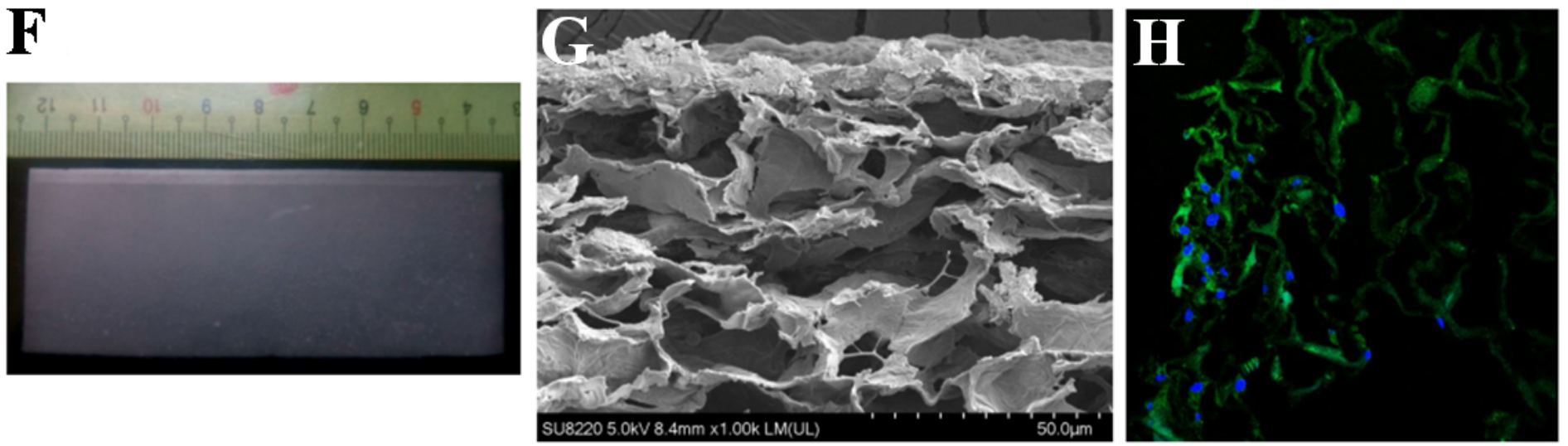

\section{Figure 3}

Differentiation of AMSCs into endothelial-like cells in alginate beads and AMSCs seeded in collagen/chitosan sheet. The results of PECAM-1 (a) and VWF (b) mRNA expression showed markedly up regulation after endothelial differentiation and increased sequentially by cultivation duration especially at days 14. (c) representative H\&E staining of alginate beads seeded with epithelial-differentiated AMSCs. The cells were evenly distributed within the porous alginate scaffold after 14 days induction. The immunofluorescent staining of PECAM-1 (d) and vWF (e) expression confirmed the capability of 
angiogenesis within the epithelial-differentiated AMSCs impregnated alginate beads. (f) representative the gross appearance and size of collagen/chitosan cell sheet. $(\mathrm{g})$ representative scanning electron microscopy analyses of the collagen/chitosan cell sheet when AMSCs were seeded and cultured for 7 days. The cell sheet microstructures showed irregular laminas with interconnected pores. The seeded AMSCs grew in a flat form from the surface to interior via pores of the cell sheet and were confirmed by the fluorescent confocal images (h). Cells were stained with F-actin (green) together with nuclear stain Hoechst 33342 (blue).
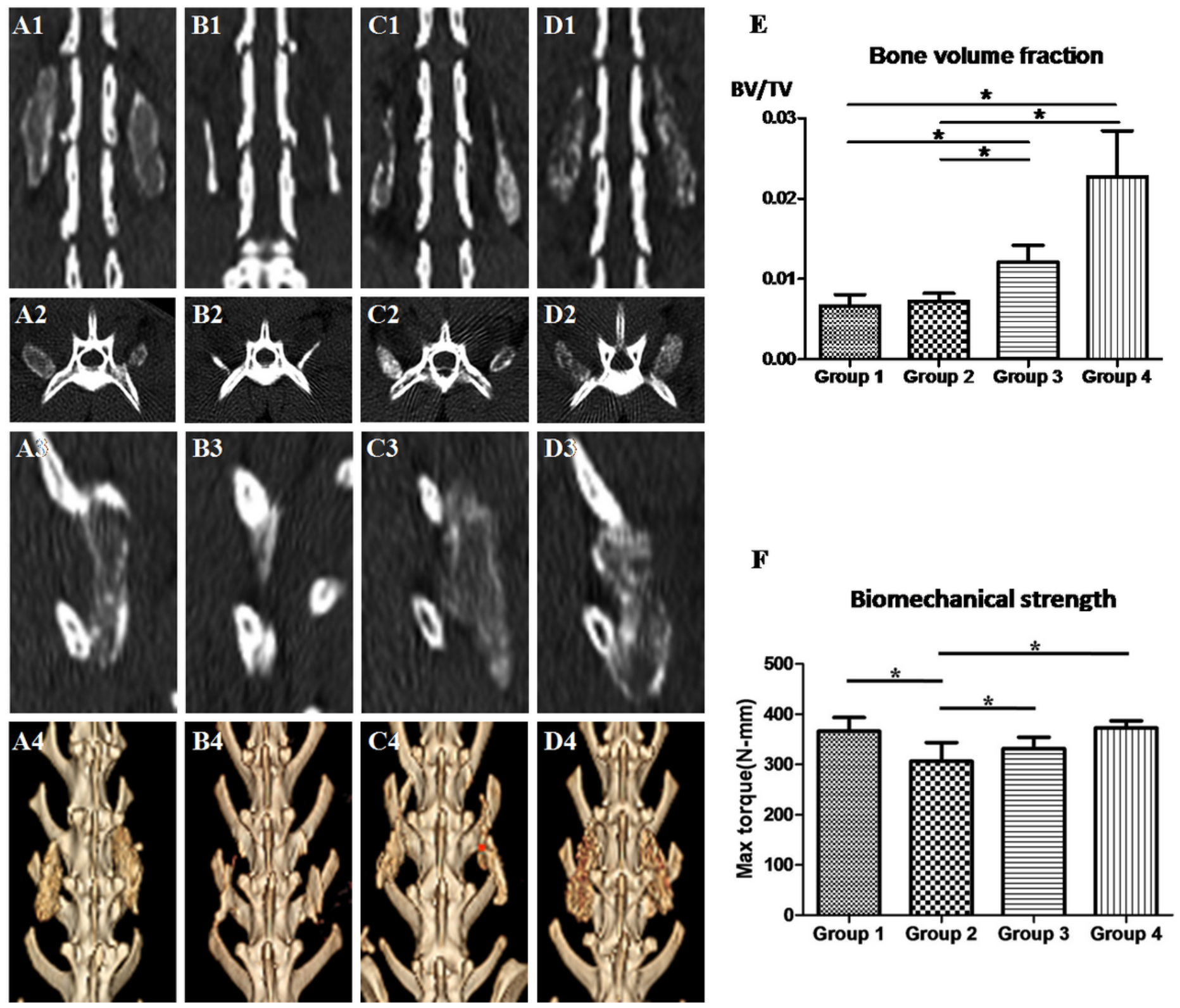

Figure 4

The results of new bone formation, ossification, and biomechanical strength at 12 weeks after surgery. Three-dimentional reconstructive coronary, axial, and sagittal computed tomography images of Group 1 (a1-4), Group 2 (b1-4), Group 3 (c1-4), and Group 4 (d1-4) illustrated bone formation in all study groups with different degree of bony ossification and continuity. Group 4 showed continuous bone mass formation with evenly distributed ossification at central and outer regions between L4- 5 transverse 
processes. (e) representative the volumetric analyses of matured bone formation and the results showed Group 4 had a tendency towards a higher volume of regenerated bone than Group $1\left({ }^{*} p<0.01\right.$ ), Group 2 ( $\left.{ }^{*} \mathrm{p}<0.01\right)$, and Group $3(\mathrm{p}=0.65)$. (f) representative the biomechanical strength analysis showed the maximal torque was significantly lower for Group 2 than the other three study groups.

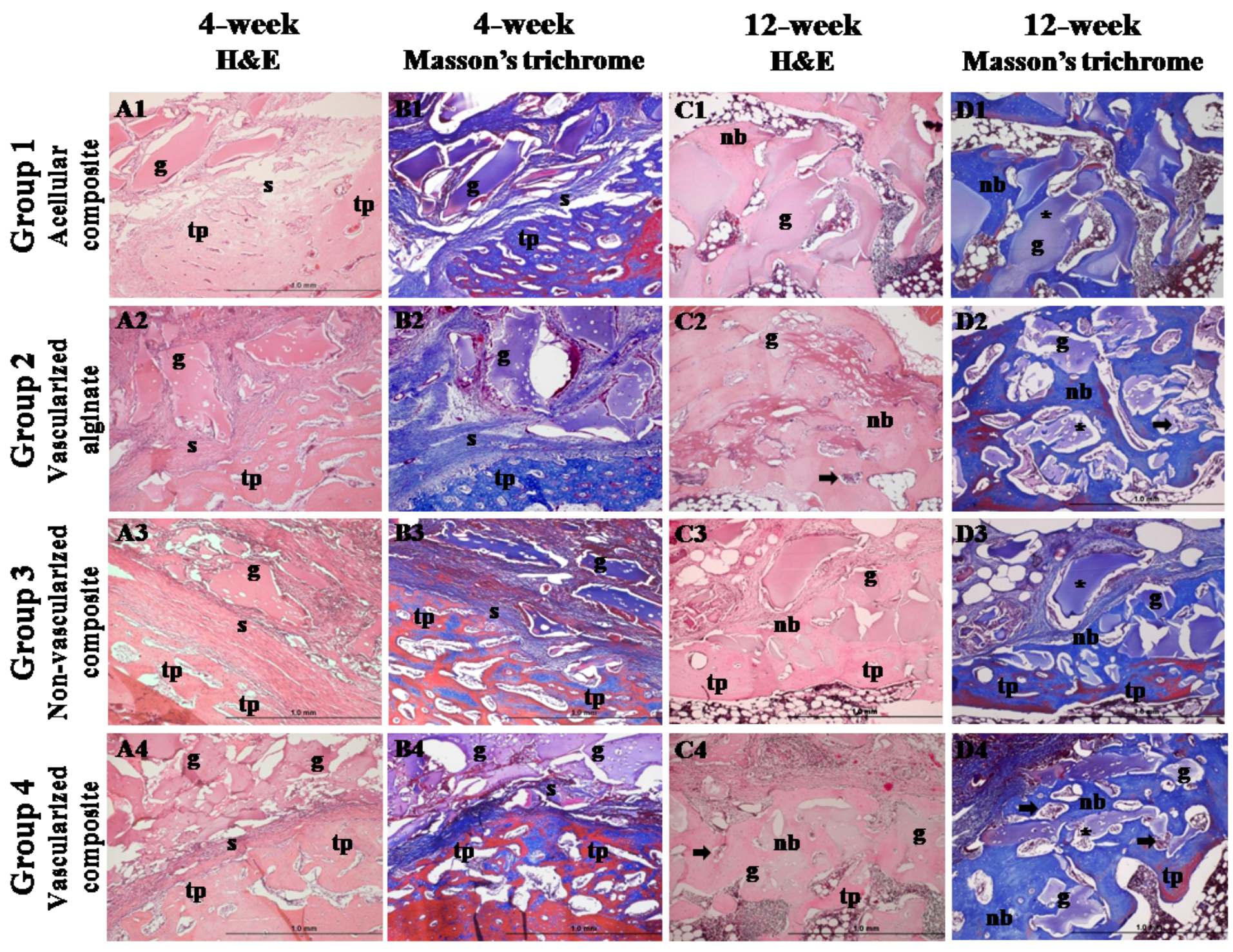

Figure 5

Time course analyses of H\&E and Masson's trichrome stained images after implantation in vivo. Except Group 1, obvious cellular micro-pores were found within the alginate beads at 4-week early stage (a1-4 and b1-4). Persistent cellular micro-pores (indicated by *) within the alginate remnants and microvascular structures (indicated by dark arrow) were observed only in Group 2 and Group 4 (c1-4 and d1-4) after 12-week implantation. Group 2 and Group 4 showed more new bone formation with thick and bridged connecting trabecular bone between alginate scaffolds (c2\&4 and d2\&4). tp= transverse processes (host bone); $\mathrm{nb}=$ new bone; $\mathrm{s}=$ collagen/chitosan sheet; $\mathrm{g}=$ residual alginate beads. 

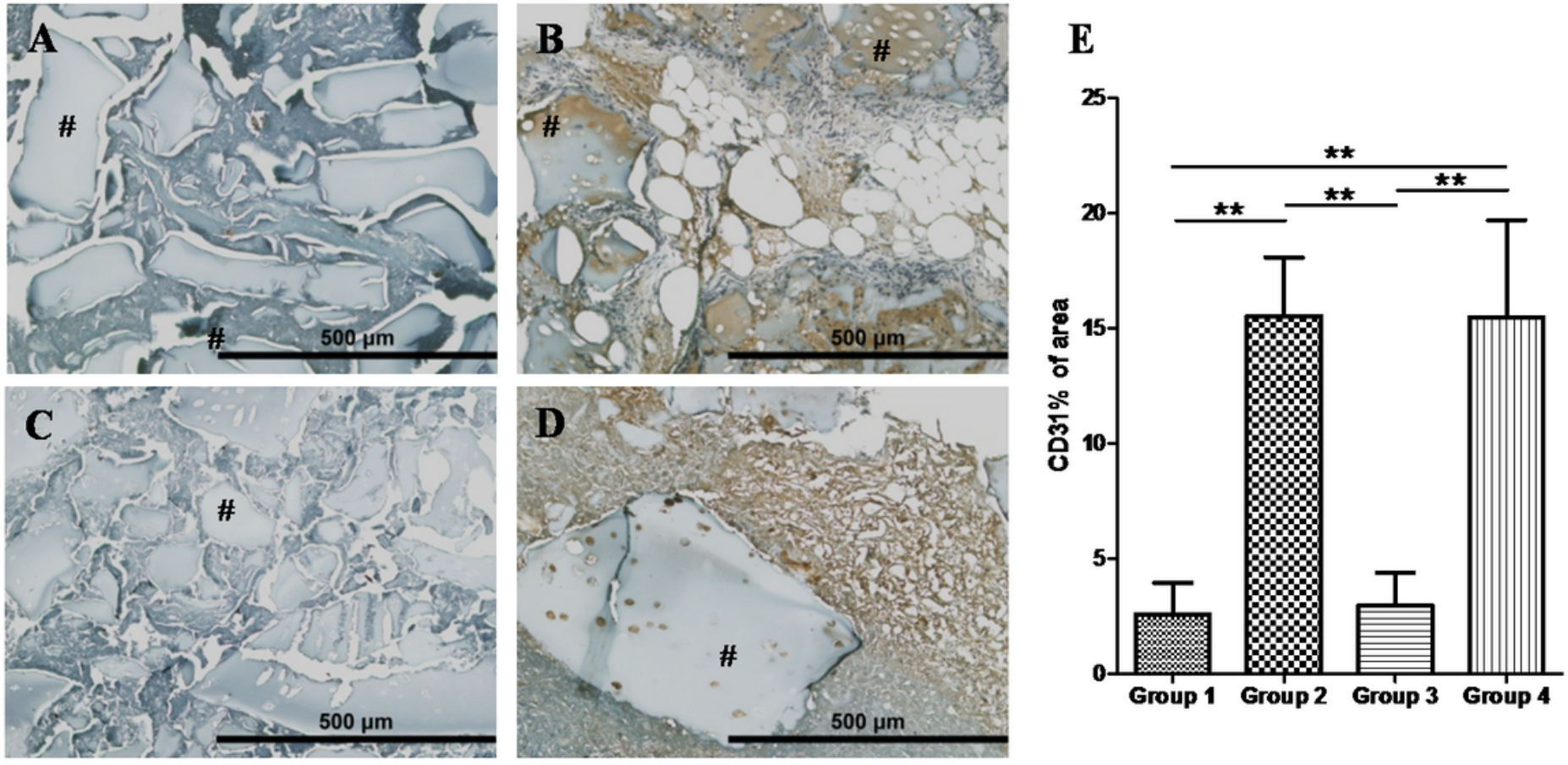

Figure 6

Immunohistochemistry stain of CD31+ blood vessel formation after 12-week implantation in vivo. Better performance of CD31+ blood vessel formation (indicated by \#) appeared for Group 2 (b) and Group 4 (d) than Group 1 (a) and Group 3 (c). Semi-quantitative analysis confirmed the image results (e). $(\star \star p<0.01)$. 